\title{
Reducing Vertical Handover Latency in IP-Based Networks
}

\author{
Ishtiaq Wahid, Ata ul Aziz Ikram, and Aftab Alam
}

\begin{abstract}
Due to extended IPv6 features, Mobile IPv6 protocol offers an improved mobility support for mobile users. Literature shows that the mobility issues in the next generation networks would be an important concern for researchers, developer and engineers. Mobility management with low latency and packet loss is a big challenge in inter technologies communication. In this study an optimized vertical handover scheme based on Hash function is proposed. The proposed model reduces the network layer registration time and address resolution time in vertical hand over process, thus the overall vertical handover latency is reduced. Further, the suggested model ensures unique Care off Address (CoA) for a mobile node, hence eliminates the Duplicate Address Detection time (DAD), which is one of the major contributors for latency in the previous vertical handover schemes.
\end{abstract}

Index Terms - Vertical handover, handover latency, mobile ipv6, 4th generation networks, seamless mobility, home agent.

\section{INTRODUCTION}

Presently, variety of wireless communication systems such as AMPS, GSM, IS-95, WCDMA, and Wireless LAN are coexisting for different services and data rates [1]. The third-generation $(3 \mathrm{G})$ systems are planned to support integrated services, such as audio, video, video conferencing and high-speed Internet connection, with the demand for real time applications. The desire for $4 \mathrm{G}$ systems is to seamlessly integrate all existing and newly developed wireless communication systems with addition to developing a new uniform standard to replace all existing technologies [2]. $4^{\text {th }}$ $\mathrm{G}$ services range from traditional voice to virtual banking, mobile office, video conferencing, and entirely audio broadcasting to watching streamed video [2]. All these services require high data rate with full mobility support. Literature shows that the mobility issues in the next generation networks would be an important concern for researchers, developer and engineers.

In $4 \mathrm{G}$ networks, there are both horizontal and vertical handoffs. Horizontal handoff manages intra-system handoff while the vertical handoff manages the inter-system handoffs. Therefore mobility management is more complex in $4 \mathrm{G}$ networks and it require more research focus, to develop

seamless mobility management protocols that ensures continuous connection to real-time high speed data and

Manuscript received October 20, 2012; revised September 10, 2012.This work was supported by Iqra University Islamabad Pakistan.

Ishtiaq Wahid is with the Iqra university, Islamabad Pakistan (e-mail: ishtiaqwahid@iqraisb.edu.pk).

Aftab Alam is with the Deptt: of Computer Science and IT University of Malakand Chakdara, KPK Pakistan (e-mail: alam@uom.edu.pk).

Ata ul Aziz Ikram is with the Deptt: of Computing and Technology at Iqra university Islamabad Pakistan (e-mail: ata@iqraisb.edu.com.). multimedia applications with low latency and packet loss.

\section{Mobile IPv6 Protocol}

As compare to MIPv4, MIPv6 provide better mobility support to its users. MIPv6 has similar principle of operations as MIPv4 but has extended features of IPv6 [3]. MIPv6 keeps communication alive while Mobile Node (MN) moves from one IPv6 sub-network to another IPv6 sub-network [2]. MIPv6 performs horizontal handoff traditionally but in vertical handoff its IP becomes invalid. The new visited network sends an advertisement message to the $\mathrm{MN}$ and creates Care off Address (CoA) for the MN. In order to keep communication between $\mathrm{MN}$ and Correspondent Node $(\mathrm{CN})$ alive, Home Agent (HA) performs binding between the $\mathrm{MN}$ home address (H@) and CoA. The home address is needed to detect packets for $\mathrm{MN}$ and the CoA is needed to locate the current position of the $\mathrm{MN}$ for the purpose of packets delivery at new location.

$\mathrm{MN}$ initiates the handoff procedure through "Neighbour discovery" scheme which is based on the advertisement of the available Access Router (AR). After link uniqueness confirmation the MN gets $\mathrm{CoA}$ with new subnet prefix in new access router (nAR). MN then exchanges its binding update information's with HA to maintain the connection. The process of MIPv6 is shown diagrammatically in Fig. 1.

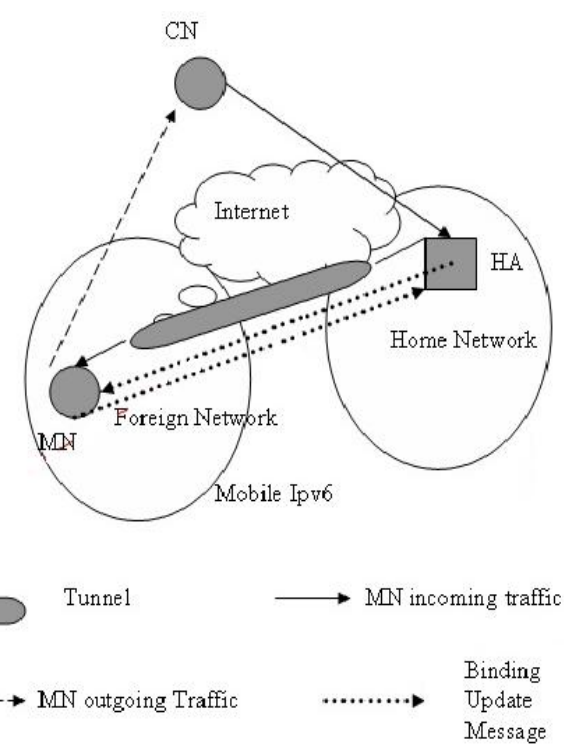

Fig. 1. MIPv6 operation [3]

This process is effective but takes time in discovering new subnet, CoA generation and binding updates, which causes latency [6]. In MIPv6 latency in handoff is given by the round trip for binding update with $\mathrm{HA}, \mathrm{CN}$ or oAR [5]. To reduce lengthy IP handover latency, two schemes based on 
Mobile IPv6, namely Hierarchal MIPv6 and Fast Handover have been proposed in the literature. Hierarchical MIPv6 aims to reduce the network registration time, and Fast-Handover attempts to minimize the address resolution delay.

\section{HIERARCHICAL MIPV6}

Hierarchical MIPv6 (HMIPv6) enhances MIPv6 by introducing hierarchal network structure [3] that reduces the registration time with HA. HA place special network agent entity at the edge of each network called Mobile Anchor Point (MAP). MAP divides the mobility into global mobility and local mobility [2]. Several AR's are collectively controlled by a single MAP which divides the global internet into regions. In regional handoff, MN gets regional CoA from nAR and shares it locally with MAP. Thus local handoff is performed by MAP with local CoA without informing the HA. In this way the HMIPv6 reduces signaling traffic and latency as shown in Fig. 2.

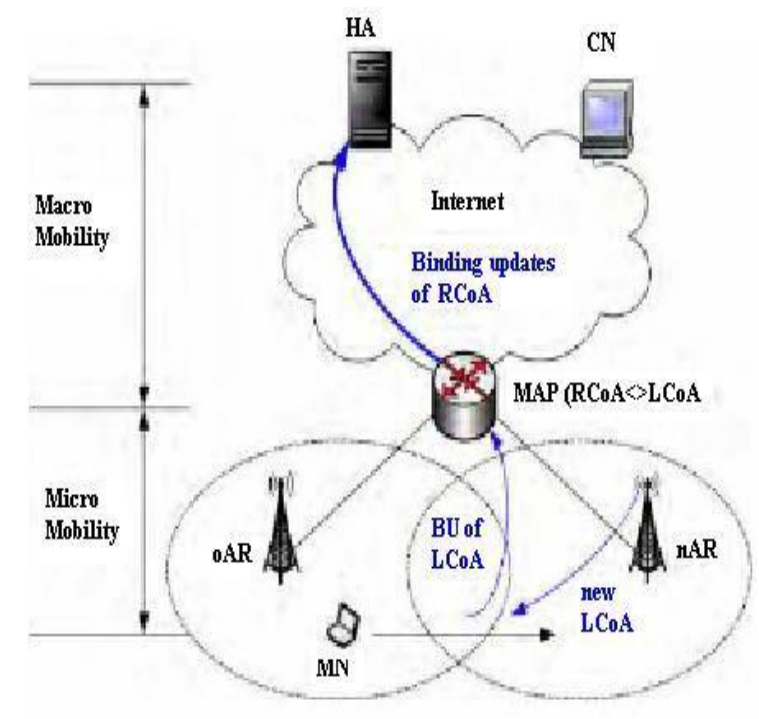

Fig. 2. HMIPv6 operations [3]

\section{FAST HANDOVER MECHANISM (FMIPV6)}

In Fast Handover the Mobile node takes the decision of performing handoff in IP-based network and updates the source router [3]. Source router asks the target router for CoA Packets for the MN are then sent to the new CoA. When the MN loses connection with source router, it instantly takes the CoA that has been pre configured for it by the nAR [4] and receives packets using CoA. Fast handover mechanism reduces address resolution time by performing pre-configuration. FMIPv6 performs its operations in three steps, handoff initiation, tunnel establishment and packets forwarding. FMIPv6 sets up a connection path between the two routers for continuous communication of the MN while handoff is in process.

\section{FAST HANDOVER MEChanism (FMIPV6) WITH HaSH}

The proposed model is a novel idea which combines the best features of Hierarchal mobile IPv6 and Fast handoff scheme with the hash algorithm. The detailed description is given below.

Registration in the proposed model will not be a 3 ways handshake process. In this model the visited network will inform the home agent or MAP that the mobile node is approaching. The remaining synchronization will be done at each side individually with the help of an algorithm which will generate the same new care off address for the Mobile node based on previous IP address. Diagrammatically it is shown in Fig. 3.

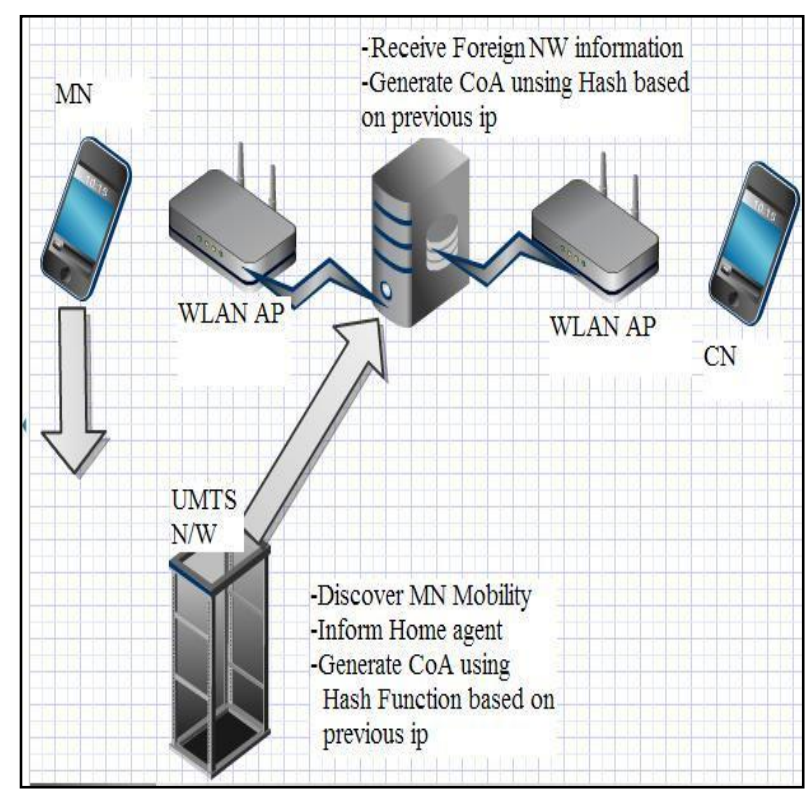

Fig. 3. Mobile Ipv6 fast handoff with hash function

\section{A. Motivation for the Proposed Scheme}

The suggested name for the proposed model is "Mobile IPv6 Fast Handoff with Hash Function"; the concept of the model is given below,

This model is inspired by the huge number of addresses in IPv6. IPv6 can theoretically hold $2^{\wedge} 128$ IP addresses. $2^{\wedge} 128=340,282,366,920,938,463,463,374,607,431,768,211$, 456

This is a very large number. Therefore a pool of IP's can be reserved in each network for clients entering due to mobility only. The next step is to design such an algorithm that will generate an IP i.e. care off address based on the previous IP for both the $\mathrm{MN}$ and Home agent. Therefore no synchronization will be needed between the MN and the Home agent for updating the IP when IP is invalidated caused by mobility to another network.

Further more the IP address generated by the Proposed algorithm, would not be given to ordinary clients i.e. these IP's would be used only for clients who are coming within range due to mobility, to reduce IP conflict. Another problem is how the algorithm will know about the network portion of the IP at the Home agent side. This could be solved by tracking the mobility of the $\mathrm{MN}$ to the neighbour network and synchronization for the network portion can be made before the actual vertical handover takes place while the remaining portion of IP can be generated automatically by the algorithm at both sides. 


\section{B. Operation of Mobile Ipv6 Fast Handover with Hash Function}

Unlike other schemes, in this model handoff process is initiated by the network as apposed to the Mobile node. By sending continuous advertisements, the router of the visited network knows about the node mobility towards its coverage area. The nAR then sends a message to the MN and HA about this mobility. After receiving message from the nAR the concern entities namely MN, HA and nAR generates the CoA of $\mathrm{MN}$ based on its previous IP address with the prefix of the new visited network using hash function. The hash algorithm generates same output every time, if it is provided with the same input. Moreover the function will generate IP in a defined range. In other words the function is bounded to produce an output from a define range of values. This eliminates the chance of IP conflict. In other schemes such as FMIPv6 after generating the CoA, it is checked for the availability which is known as Duplicate Address Detection (DAD). The DAD time increases the overall latency of the handoff process. The proposed model ensures no IP conflict, so DAD is completely eliminated, hence overall latency is reduced. All this process will be completed before actual handoff process starts. In other words the model is performing the FMIPv6 operation in an enhanced mode up to this point.

In the second step when the signal strength of $\mathrm{MN}$ reaches its threshold value it just sends an acknowledgement for the Handoff. All the concerned entities have the CoA address in advance, so they just start communication with the new care off address (nCoA) of the MN. Diagrammatically this process is explained below in Fig. 4.

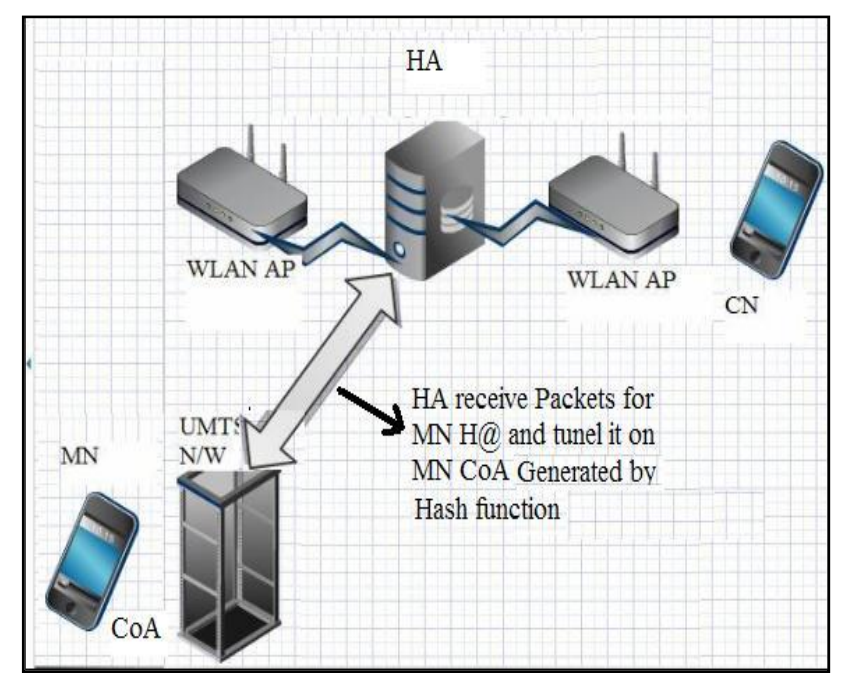

Fig. 4. Mobile Ipv6 fast handoff with hash function

\section{Features of FMIPv6 with Hash}

- The proposed model has the following distinguishing features.

- It ensures a unique IP and eliminates DAD.

- It reduces address resolution time.
- It reduces traffic signalling by eliminating binding update messages (The registration traffic signalling is minimized to router advertisement and acknowledgement only).

\section{Model Limitations}

This model proposes a new infrastructure which needs validation and requires simulation. This article is not touched upon implementation, and more research is required for implementation of algorithms.

\section{CONCLUSION}

The proposed model has the capability to reduce latency and is more efficient then the previous models. The model has features both of the HMIPv6 and FMIPv6 with added capability of eliminating DAD by ensuring unique IP and reducing traffic signalling by minimizing the binding update traffic.

\section{REFERENCES}

[1] N. Nakjima and Y. Yamao, "Development of 4th Generation Mobile Communication," Wireless Commun. and Mobile Comp., vol. 1, no. 1. 2001.

[2] C. Liu and C. Zhou "Challenges and Solutions for Handoff Issues in 4G Wireless Systems an Overview,” LACCEI, 2004.

[3] C.-K. Lau, "Improving Mobile IP Handover Latency on End-to-End TCP in UMTS/WCDMA Networks," School of Electrical Engineering and Telecommunications, The University of New South Wales March, 2006

[4] R. Koodli, "Fast Handovers for Mobile IPv6," IETF, March 2003.

[5] X. P. Costa, R. Schmitz, and H. Hartenstein, "A MIPv6, FMIPv6 and HMIPv6 handover latency study: analytical approach Marco Liebsch Network Laboratories," Adenauerplatz 6, NEC Europe Ltd., 69115 Heidelberg, Germany.

[6] R. Koodli, Mobile IPv6 Fast Handovers, Request for Comments: 5268 Starent Networks Obsoletes: 4068 June 2008

Ishtiaq Wahid is an IEEE student member. He received his B.S. degree in Information Technology from University of Malakand at Chakdara, Dir lower, KPK, Pakistan, in 2007 and the M.S. degree in Computer Science from Iqra University Islamabad Pakistan in 2009. He is currently pursuing the Ph.D. degree with Department of Computing in Technology Iqra University Islamabad Pakistan. In 2010, he joined in University of Malakand as a lecturer. Since 2010, he has been a lecturer with this Institute. His current research interests include Ad-hoc networks, wireless communications, and virtual reality environment.

Ataul Aziz Ikram is the Head of Department of computing and technology, Iqra university Islamabad. He did his Ph.D. in Electrical Engineering from City University of New York, M.Phil in Electrical Engineering from City University of New York, M.S. in Electrical Engineering from City University of New York, and B.S. in Electrical and Electronics Engineering; from Middle East Technical University, Ankara, Turkey. His areas of interest are Evolving wireless technologies, Cross Layer Design, Heterogeneous Networks, Next Generation Wireless Technologies, Physical Layer Testbeds, MIMO-OFDMA, UWB, Semiconductor Device Design and Fabrication for different applications.

A. Aftab Alam was born in 1982, in Chakdara, Pakistan. He received his BS-IT (Hons) degree from University Of Malakand, Pakistan in 2007 and MS(CS) degree from FSAT-NU, Pakistan in 2010, with specialization in Networking. Currently he is perusing his Ph.D from University of Malakand, Pakistan. His current research interests include information security, next generation networks, 3D Graphics and Bioinformatics. 